\title{
ON BANACH SPACE PROJECTIVE TENSOR PRODUCT OF $C^{*}$-ALGEBRAS
}

\author{
VED PRAKASH GUPTA AND RANJANA JAIN
}

\begin{abstract}
We analyze certain algebraic structures of the Banach space projective tensor product of $C^{*}$-algebras which are comparable with their known counterparts for the Haagerup tensor product and the operator space projective tensor product of $C^{*}$-algebras. Highlights of this analysis include (a) injectivity of the Banach space projective tensor product when restricted to the tensor products of $C^{*}$-algebras, (b) detailed structure of closed ideals of $A \otimes^{\gamma} B$ in terms of those of $A$ and $B$, (c) identification of certain spaces of ideals of $A \otimes^{\gamma} B$ in terms of those of $A$ and $B$ from the perspective of hull-kernel topology, and (d) identification of the center of $A \otimes^{\gamma} B$ with $\mathcal{Z}(A) \otimes^{\gamma} \mathcal{Z}(B)$, where $A$ and $B$ are $C^{*}$-algebras.
\end{abstract}

\section{INTRODUCTION}

Around 1960s, Gelbaum initiated the study of certain spaces of ideals of the Banach space projective tensor product $A \otimes^{\gamma} B$ of Banach algebras $A$ and $B$, who was then followed by Laursen and Tomiyama - see $9,10,11,23,29$ and the references therein. They focussed mainly on analyzing the spaces of maximal modular ideals and primitive ideals of $A \otimes^{\gamma} B$ in terms of those of $A$ and $B$, and to analyze which properties of Banach algebras are passed on to their tensor products, where $A$ or $B$ or both is/are commutative. However, not much was discovered about the structure of general ideals of $A \otimes^{\gamma} B$ in terms of those of $A$ and $B$. A similar hardship has been observed in understanding the ideal structure of $A \otimes{ }^{\min } B$ as well, for $C^{*}$-algebras $A$ and $B$.

On the other hand, the analysis of various algebraic structures of the Haagerup tensor product $A \otimes^{h} B$ and the operator space projective tensor product $A \widehat{\otimes} B$, of $C^{*}$-algebras $A$ and $B$, has been carried out extensively during last three decades (see [1, 4, 15, 16, 17, 18, 20, 21, 22]). An important and useful development of this project has been the discovery of the connection that exists between the structures of centers and ideals of $A \otimes^{h} B$ and $A \widehat{\otimes} B$ in terms of those of $A$ and $B$, all of which was pioneered by the remarkable work [1] of Allen, Sinclair and Smith, wherein they study closed ideals of $A \otimes^{h} B$. This article aims at obtaining similar results for $A \otimes^{\gamma} B$, for $C^{*}$-algebras $A$ and $B$.

A closer look reveals that a crucial ingredient that helped to establish the relationships mentioned above was the injectiviy of $\otimes^{h}$ and a partial injectivity of $\widehat{\otimes}$ (see Proposition 2.1. below), along with an exactness type property (as in Proposition 3.10) exhibited by both tensor norms. It is known that $\otimes^{\gamma}$ is not injective; and, might be due to the lack of any partial injectivity result, not much could be known about the algebraic structure of $A \otimes^{\gamma} B$. However, exploiting a work of Diestel et al. [6], the second named author had demonstrated in her Ph.D. thesis 14 that, when restricted to the tensor products of $C^{*}$-algebras, $\otimes^{\gamma}$ observes a better partial injectivity than $\widehat{\otimes}$, as is re-produced in Section 2 below. This turns out to have beautiful

2010 Mathematics Subject Classification. 46L06,46M05,46H10.

Key words and phrases. Banach $*$-algebras, $C^{*}$-algebras, Banach space projective tensor product, *subalgebras and ideals.

The first named author was supported by a UPE-II project (with Id 228) of Jawaharlal Nehru University, New Delhi. 
consequences in the study of algebraic structures of $A \otimes^{\gamma} B$, which is achieved primarily by employing a similar line of treatment as in [1. We present here few of those consequences and are quite hopeful that we can deduce more.

For any algebra $A$, let $\mathcal{M}(A)$ (resp., $\mathcal{M}_{m}(A)$ ) denote the set of maximal ideals (resp., maximal modular ideals) of $A$. In his first article to analyze spaces of ideals of $A \otimes^{\gamma} B$, Gelbaum ([9]) showed that if $A$ and $B$ are commutative Banach algebras, then there is a homeomorphism between $\mathcal{M}_{m}(A) \times \mathcal{M}_{m}(B)$ and $\mathcal{M}_{m}\left(A \otimes^{\gamma} B\right)$, when the spaces are equipped with $w^{*}$-topologies. Then, in 11, he replaced the condition of commutativity by existence of unity and obtained an injective map from $\mathcal{M}(A) \times \mathcal{M}(B)$ into $\mathcal{M}\left(A \otimes^{\gamma} B\right)$ which turned out to be closed and continuous with respect to the hull-kernel topology. Surjectivity is still an open question in this case. Almost simultaneously, Laursen ([23]) dropped the commutativity of one of the Banach algebras and established that the spaces $\mathcal{M}_{m}(A) \times \mathcal{M}_{m}(B)$ and $\mathcal{M}_{m}\left(A \otimes^{\gamma} B\right)$ are homeomorphic with respect to hull-kernel topology. For arbitrary $C^{*}$-algebras $A$ and $B$ (not necessarily unital or commutative), based on the ideal structue of $A \otimes^{\gamma} B$ discussed above, we establish that there is a homeomorphism from $I d^{\prime}(A) \times I d^{\prime}(B)$ onto its image (which is also dense) in $I d^{\prime}\left(A \otimes^{\gamma} B\right)$, with respect to $\tau_{w}$-topology, where $I d^{\prime}(A)$ denotes the set of proper closed ideals of $A$. Moreover, this map restricts to a homeomorphism from $\mathcal{M}_{m}(A) \times \mathcal{M}_{m}(B)($ resp., $\mathcal{M}(A) \times \mathcal{M}(B))$ onto $\mathcal{M}_{m}\left(A \otimes^{\gamma} B\right)$ (resp., $\mathcal{M}\left(A \otimes^{\gamma} B\right)$ )with respect to the hull-kernel topology.

Here is a brief overview of the topics discussed in this article. As mentioned above, Section 2 is devoted towards establishing a partial injectivity of $\otimes^{\gamma}$ when restricted to tensor products of $C^{*}$-algebras, which is used throughout the article. Section 3 is the soul of this article which provides a thorough discussion of ideal structure of $A \otimes^{\gamma} B$ in terms of ideals of $C^{*}$-algebras $A$ and $B$. Among other things, we show that every closed ideal of $A \otimes^{\gamma} B$ contains a product ideal; that if $A$ or $B$ has finitely many closed ideals, then every closed ideal of $A \otimes^{\gamma} B$ is a sum of product ideals, and we identify minimal (resp., maximal and maximal modular) ideals of $A \otimes^{\gamma} B$ in terms of those of $A$ and $B$. In Section 4, we identify spaces of proper (resp., maximal and maximal modular) ideals of $A \otimes^{\gamma} B$ in terms of those of $A$ and $B$ from the perspective of hull-kernel topology. Finally, Section 5 provides another application of partial injectivity of $\otimes^{\gamma}$, where we provide an identification of the center of $A \otimes^{\gamma} B$ with $\mathcal{Z}(A) \otimes^{\gamma} \mathcal{Z}(B)$.

\section{InjeCtivity of the BANACH SPACE PROJECTIVE NORM ON TENSOR PRODUCTS OF $C^{*}$-ALGEBRAS}

Recall that, a norm $\|\cdot\|_{\alpha}$ on the algebraic tensor product $A \otimes B$ of a pair of $C^{*}$-algebras $A$ and $B$ is said to be

(1) a cross norm if $\|a \otimes b\|_{\alpha}=\|a\|\|b\|$ for all $a \in A, b \in B$,

(2) an algebra norm if $\|w z\|_{\alpha} \leq\|w\|_{\alpha}\|z\|_{\alpha}$ for all $w, z \in A \otimes B$, and

(3) a tensor norm if $\|\cdot\|_{\lambda} \leq\|\cdot\|_{\alpha} \leq\|\cdot\|_{\gamma}$, where $\lambda$ and $\gamma$ are the Banach space injective and projective norms, respectively.

Clearly, $A \otimes^{\alpha} B$, the completion of $A \otimes B$ with respect to any algebra norm $\|\cdot\|_{\alpha}$, is a Banach algebra. From an algebraic point of view, among the well analyzed tensor products in literature are the $C^{*}$-minimal tensor product $\left(\otimes^{\mathrm{min}}\right)$, the Haagerup tensor product $\left(\otimes^{h}\right)$, the operator space projective tensor product $(\widehat{\otimes})$ and the Banach space projective tensor product $\left(\otimes^{\gamma}\right)$. We refer the reader to [8, 28, and [27] for their definitions and essential properties. All these norms are cross algebra tensor norms and yield Banach algebras. In fact, for $C^{*}$-algebras $A$ and $B$, the canonical involution on $A \otimes B$ is not isometric 1 with respect to $\otimes^{h}$ (see [1]); however, by the very definition of $\|\cdot\|_{\gamma}, A \otimes^{\gamma} B$ is a Banach *-algebra; and, by [19], so is $A \widehat{\otimes} B$.

\footnotetext{
${ }^{1}$ We follow the convention that the involution on a Banach $*$-algebra is an isometry.
} 
The $C^{*}$-minimal tensor product is known to be injective and so is the Haagerup tensor product (see [1, 8]). On the other hand, neither the Banach space projective tensor product nor the operator space projective tensor product is injective. But in few cases they behave well. For instance, Kumar ([19]) proved that the tensor product of ideals of $C^{*}$-algebras can still be embedded nicely.

Proposition 2.1. (19, Theorem 5]) Let $A$ and $B$ be $C^{*}$-algebras, and $I$ and $J$ be closed ideals in $A$ and $B$, respectively. Then the identity map on $I \otimes J$ extends to an isometric algebra map from the Banach algebra $I \widehat{\otimes} J$ onto the product ideal $\overline{I \otimes J} \subseteq A \widehat{\otimes} B$.

The situation with the Banach space projective tensor product of $C^{*}$-algebras is even better (Theorem 2.6 below) as was proved in the doctoral thesis [14] of the second named author. Given its usefulness, we include the details for convenience of present and future references.

We first recall some concepts whose details can be found in [6] and the references therein. The strong*-topology on a Banach space $X$ is defined to be the locally convex topology generated by the seminorms $x \mapsto\|T x\|$ for bounded linear maps $T$ from $X$ into Hilbert spaces. Also, an operator $T: A \rightarrow X$, where $A$ is a $C^{*}$-algebra and $X$ is a Banach space, is said to be $p$ - $C^{*}$ summing for $p>0$ if there exists a constant $k$ such that for every finite sequence $\left(a_{1}, a_{2}, \ldots, a_{n}\right)$ of self-adjoint elements in $A$,

$$
\left(\sum_{i=1}^{n}\left\|T\left(a_{i}\right)\right\|^{p}\right)^{1 / p} \leq k\left\|\left(\sum_{i=1}^{n}\left|a_{i}\right|^{p}\right)^{1 / p}\right\|,
$$

where for $a \in A,|a|:=\left(\frac{a a^{*}+a^{*} a}{2}\right)^{1 / 2}$. We first collect few useful results.

Proposition 2.2. [6, §3]: For a $C^{*}$-algebra $A$ and Banach space $X$, an operator $T: A \rightarrow X$ is 2- $C^{*}$-summing if and only if it is strong*-norm continuous.

Theorem 2.3. [13, Proposition 2.1] Every bounded linear map $T: A \rightarrow B^{*}$, $A$ and $B$ being $C^{*}$-algebras, can be factored through a Hilbert space, that is, there exists a Hilbert space $H$, and bounded linear maps $u: A \rightarrow H$ and $v: H \rightarrow B^{*}$ such that $T=v \circ u$.

Theorem 2.4. [6, Theorem 3.2] Let $T: X \rightarrow Y$ be a bounded linear operator between Banach spaces $X$ and $Y$. Then $T$ is strong ${ }^{*}$-norm continuous if and only if $T$ factors through a Hilbert space.

Theorem 2.5. 6. Theorem 3.6] Let $A$ be a $C^{*}$-algebra, $B$ be a $C^{*}$-subalgebra of $A$ and $Y$ be any Banach space. Then every 2- $C^{*}$-summing operator $T: B \rightarrow Y$ extends to a norm preserving $2-C^{*}$-summing operator $\widetilde{T}: A \rightarrow Y$.

Now, with these results in hand, we are ready to prove our result regarding the injectivity of Banach space projective norm in the $C^{*}$ set-up.

TheOREM 2.6. Let $A_{1}$ and $B_{1}$ be $C^{*}$-subalgebras of $C^{*}$-algebras $A$ and $B$, respectively. Then the identity map on $A_{1} \otimes B_{1}$ extends to an isometric *-algebra map from $A_{1} \otimes \gamma B_{1}$ onto the closed *-subalgebra $\overline{A_{1} \otimes B_{1}} \subseteq A \otimes \otimes^{\gamma} B$.

Proof. By [27, Corollary 2.12], $A_{1} \otimes^{\gamma} B_{1}$ is a subspace of $A \otimes^{\gamma} B_{1}$ if and only if every bounded operator from $A_{1}$ into $B_{1}^{*}$ extends to an operator of the same norm from $A$ into $B_{1}^{*}$. Let $T$ be one such operator from $A_{1}$ into $B_{1}^{*}$. Then, by Theorem 2.3. $T$ can be factored through a Hilbert space, which, by Theorem 2.4 is strong*-norm continuous. By Proposition 2.2. $T$ is $2-C^{*}$-summing, therefore, by Theorem 2.5. $T$ extends to a norm preserving 2- $C^{*}$-summing operator $\tilde{T}: A \rightarrow B_{1}^{*}$. Thus $A_{1} \otimes^{\gamma} B_{1}$ is a closed subspace of $A \otimes^{\gamma} B_{1}$. Since $\otimes^{\gamma}$ is symmetric, it also follows that $A \otimes^{\gamma} B_{1}$ is a closed subspace of $A \otimes^{\gamma} B$. 
V. P. GUPTA AND R. JAIN

\section{Ideal structure of $A \otimes^{\gamma} B$}

If $\alpha$ is either the Haagerup tensor product or the operator space projective tensor product, $A$ and $B$ are $C^{*}$-algebras with $A$ topologically simple, then by [1, Proposition 5.2], and by [17. Theorem 3.8], it is known that every closed ideal of the Banach algebra $A \otimes^{\alpha} B$ is a product ideal of the form $A \otimes^{\alpha} J$ for some closed ideal $J$ in $B$. For $\otimes^{\min }$, we obtained, in [12, the following analogue of above results:

Theorem 3.1. [12] Let $A$ and $B$ be $C^{*}$-algebras where $A$ is topologically simple. If either $A$ is exact or $B$ is nuclear, then every closed ideal of the $C^{*}$-algebra $A \otimes^{\min } B$ is a product ideal of the form $A \otimes^{\min } J$ for some closed ideal $J$ in $B$.

It turns out that the ideal structure of the Banach space projective tensor product $A \otimes^{\gamma} B$ also follows on similar lines as in Theorem 3.1 provided its ingredients can be established - see Theorem 3.13 below. The steps involved are very much on the lines of [1] and [17, and we avoid mentioning them at every instance.

Since $\|\cdot\|_{\min }$ and $\|\cdot\|_{h}$ are cross-norms and $\|\cdot\|_{\lambda} \leq\|\cdot\|_{\min } \leq\|\cdot\|_{h} \leq\|\cdot\|_{\gamma}$, where $\|\cdot\|_{\lambda}$ is the Banach space injective tensor norm, by the Remark on Page 97 of [13, we have the following crucial embeddings.

Proposition 3.2. 13 Let $A$ and $B$ be $C^{*}$-algebras. Then the identity map on $A \otimes B$ extends to a contractive injective $*$-homomorphism $i_{\min }: A \otimes^{\gamma} B \rightarrow A \otimes{ }^{\min } B$ and injective homomorphisms $i_{h}: A \otimes^{\gamma} B \rightarrow A \otimes^{h} B$ and $j_{\min }: A \otimes^{h} B \rightarrow A \otimes^{\min } B$. Also, the diagram

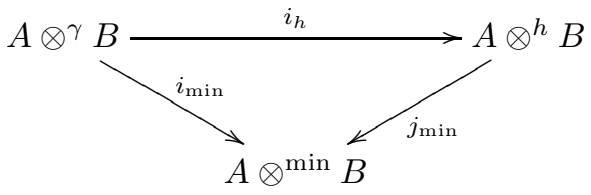

commutes.

For a closed ideal $I$ in $A \otimes^{\gamma} B$, let $I_{\min }:=\overline{i_{\min }(I)} \subseteq A \otimes^{\min } B$. On the lines of 11, Lemma 4.2], Kumar and Rajpal 20] proved the following useful result.

Proposition 3.3. 20] Let $M$ and $N$ be von Neumann algebras and let $I$ be a closed ideal in $M \otimes^{\gamma} N$. If $1 \otimes 1 \in I_{\min } \subseteq M \otimes^{\min } N$, then $1 \otimes 1 \in I$, and, in particular, I equals $M \otimes^{\gamma} N$.

Analogous to [1, Theorem 4.4], we now prove a theorem that, along with Theorem 2.6, turns out to be the main ingredient in the study of ideal structure of Banach space projective tensor product of $C^{*}$-algebras.

Theorem 3.4. Let $A$ and $B$ be $C^{*}$-algebras and let $I$ be a closed ideal in $A \otimes^{\gamma} B$. If an elementary tensor $a \otimes b \in I_{\min }$, then $a \otimes b \in I$.

Proof. We first prove for $a, b \geq 0$. Suppose $a \otimes b \in I_{\min }$ and is not in $I$. By Hahn-Banach Theorem, there exists a $\varphi \in\left(A \otimes^{\gamma} B\right)^{*}$ such that $\varphi(I)=(0)$ and $\varphi(a \otimes b) \neq 0$. It is well known that $\left(A \otimes^{\gamma} B\right)^{*}$ can be identified canonically with $B\left(A, B^{*}\right)$, the space of bounded linear maps from $A$ into $B^{*}$ - see [27, $\S 2$, page 24]. In particular, there exists a $\Phi \in B\left(A, B^{*}\right)$ such that $\varphi(x \otimes y)=\Phi(x)(y)$ for all $x \in A$ and $y \in B$. Note that $\Phi^{* *}: A^{* *} \rightarrow B^{* * *}$ is $w^{*}$-w continuous and satisfies $\left\|\Phi^{* *}\right\|=\|\Phi\|=\|\varphi\|$. Further, the association $A^{* *} \otimes B^{* *} \ni u \otimes v \mapsto \Phi^{* *}(u)(v) \in \mathbb{C}$ extends linearly to a continuous functional on $A^{* *} \otimes^{\gamma} B^{* *}$, say, $\tilde{\varphi}$. Since $A \otimes^{\gamma} B \subseteq A^{* *} \otimes^{\gamma} B^{* *}$ (27, Corollary 2.14] or Theorem 2.6) , $\tilde{\varphi}$ extends $\varphi$ and also $\|\tilde{\varphi}\|=\|\varphi\|$.

Now, consider the enveloping von Neumann algebras $M:=A^{* *}, N:=B^{* *}$, and let $\tilde{I}$ be the closed ideal in $M \otimes^{\gamma} N$ generated by $I$. We claim that $\tilde{\varphi}(\tilde{I})=(0)$ as well. For this, it is enough 
to show that

$$
\tilde{\varphi}((u \otimes s) z(v \otimes t))=0
$$

for all $z \in I, u, v \in M$ and $s, t \in N$. To begin with, let $z \in I \subseteq A \otimes^{\gamma} B, u \in M, v \in A$ and $s, t \in B$. By [27, Proposition 2.8], there exist bounded sequences $\left\{a_{i}\right\} \subset A$ and $\left\{b_{i}\right\} \subset B$ such that $z=\sum_{i=1}^{\infty} a_{i} \otimes b_{i}$. For each $n \geq 1$, consider $\omega_{n} \in M^{*}$ given by

$$
\omega_{n}(x)=\sum_{i=1}^{n} \Phi^{* *}\left(x a_{i} v\right)\left(s b_{i} t\right), x \in M .
$$

Since $\Phi^{* *}$ is $w^{*}-w^{*}$ continuous, $\omega_{n}$ is $w^{*}$-continuous, i.e., $\omega_{n} \in M_{*}$, the predual of $M$, for all $n \geq 1$. Also, for $m<n$, we observe that

$$
\begin{aligned}
\left\|\omega_{n}(x)-\omega_{m}(x)\right\| & =\left\|\tilde{\varphi}\left((x \otimes s)\left(\sum_{i=m+1}^{n} a_{i} \otimes b_{i}\right)(v \otimes t)\right)\right\| \\
& \leq\|\varphi\|\|x\|\|s\| \sum_{i=m+1}^{n} a_{i} \otimes b_{i}\left\|_{\gamma}\right\| v\|\| t \|
\end{aligned}
$$

for all $x \in M$. In particular, $\left\{\omega_{n}\right\}$ is a Cauchy sequence in $M^{*}$ with a limit, say, $\omega \in M^{*}$. Given the actions of $\omega_{n}$ 's on $M$, the obvious candidate for $\omega$ is given by $\omega(x)=\sum_{i=1}^{\infty} \Phi^{* *}\left(x a_{i} v\right)\left(s b_{i} t\right)$ for all $x \in M$. In particular, $\omega \in M_{*}$. Since, $\varphi(I)=0$ and $z \in I$, we easily see that $\sum_{i=1}^{\infty} \varphi\left(x a_{i} v \otimes\right.$ $\left.s b_{i} t\right)=0$ for all $x \in A$. This implies that $A \subseteq \operatorname{ker}(\omega)$ and, since $\omega$ is $w^{*}$ - (equivalently, $\sigma$-weakly) continuous, by von Neumann's Bicommutant Theorem, we obtain $M=A^{* *}=\bar{A}^{w^{*}}=\operatorname{ker}(\omega)$, i.e., Equation (3.2) holds for all $u \in M, v \in A$ and $s, t \in B$. Repeating the argument by letting $v, s$ and $t$ vary successively, we conclude that Equation (3.2) holds for all $z \in I, u, v \in$ $M$ and $s, t \in N$.

With this observation at our disposal, we now show that $a \otimes b$ can be approximated appropriately by elements of $\tilde{I}$ and deduce that it is annihilated by $\tilde{\varphi}$ to obtain a contradiction.

For each $\epsilon, \nu>0$, let $p_{\epsilon}, q_{\nu}$ be the spectral projections in $M$ and $N$ associated to $a$ and $b$ for the closed intervals $[\epsilon, \infty)$ and $[\nu, \infty)$, respectively. Then $p_{\epsilon} M p_{\epsilon}$ and $q_{\nu} N q_{\nu}$ are von Neumann subalgebras of $M$ and $N$ with units $p_{\epsilon}$ and $q_{\nu}$, respectively. In view of the embedding given in Theorem [2.6. consider the closed ideal $\tilde{I}_{\epsilon, \nu}:=\tilde{I} \cap\left(p_{\epsilon} M p_{\epsilon} \otimes^{\gamma} q_{\nu} N q_{\nu}\right)$. We claim that $\left(\tilde{I}_{\epsilon, \nu}\right)_{\min } \subseteq p_{\epsilon} M p_{\epsilon} \otimes^{\min } q_{\nu} N q_{\nu}$ contains the unit $p_{\epsilon} \otimes q_{\nu}$. This will then, by Proposition 3.3 . yield $\tilde{I}_{\epsilon, \nu}=p_{\epsilon} M p_{\epsilon} \otimes^{\gamma} q_{\nu} N q_{\nu}$ implying that $p_{\epsilon} a \otimes q_{\nu} b \in \tilde{I}_{\epsilon, \nu}$. In particular, $p_{\epsilon} a \otimes q_{\nu} b \in \tilde{I}$ for all $\epsilon, \nu>0$. And since $p_{\epsilon} a \stackrel{w^{*}}{\rightarrow} a$ in $M$ and $q_{\nu} b \stackrel{w^{*}}{\rightarrow} b$ in $N$, we will obtain $\tilde{\varphi}(a \otimes b)=$ $\lim _{\epsilon \rightarrow 0} \lim _{\nu \rightarrow 0} \tilde{\varphi}\left(p_{\epsilon} a \otimes q_{\nu} b\right)=0$, giving the desired contradiction.

Towards the claim, note that, by bounded functional calculus, $p_{\epsilon} a$ and $q_{\nu} b$ are invertible in $p_{\epsilon} M p_{\epsilon}$ and $q_{\nu} N q_{\nu}$, respectively. If $\left\{z_{n}\right\}$ is a sequence in $I$ such that $\left\{i\left(z_{n}\right)\right\}$ converges to $a \otimes b$ in $I_{\min } \subseteq A \otimes{ }^{\min } B \subset M \otimes \otimes^{\min } N$, then, again by Theorem 2.6, the sequence $\left\{\left(p_{\epsilon} \otimes q_{\nu}\right) z_{n}\left(p_{\epsilon} \otimes q_{\nu}\right)\right\}$ is contained in $\tilde{I}_{\epsilon, \nu}$ and $j\left(\left(p_{\epsilon} \otimes q_{\nu}\right) z_{n}\left(p_{\epsilon} \otimes q_{\nu}\right)\right) \rightarrow p_{\epsilon} a \otimes q_{\nu} b$ in $p_{\epsilon} M p_{\epsilon} \otimes{ }^{\min } q_{\nu} N q_{\nu} \subseteq M \otimes{ }^{\min } N$, where $j$ is the injective homomorphism from $p_{\epsilon} M p_{\epsilon} \otimes \gamma q_{\nu} N q_{\nu}$ into $p_{\epsilon} M p_{\epsilon} \otimes{ }^{\text {min }} q_{\nu} N q_{\nu}$ guaranteed by Proposition 3.2 . This shows that the invertible element $p_{\epsilon} a \otimes q_{\nu} b$ belongs to $\left(\tilde{I}_{\epsilon, \nu}\right)_{\min }$ and hence the unit $p_{\epsilon} \otimes q_{\nu}$ also belongs to $\left(\tilde{I}_{\epsilon, \nu}\right)_{\min }$.

Finally, for arbitrary $a$ and $b$, if $a \otimes b \in I_{\min }$ then, using above positive case, on the lines of last part of proof of [1, Theorem 4.4], it can be shown that $a \otimes b \in I$.

Corollary 3.5. Let $A$ and $B$ be $C^{*}$-algebras and let $I$ be a closed ideal in $A \otimes^{\gamma} B$. If an elementary tensor $a \otimes b \in I_{h}:={\overline{i_{h}(I)}}^{h} \subset A \otimes \otimes^{h} B$, then $a \otimes b \in I$. 
Proof. By Equation (3.1), we have ${\overline{j_{\min }\left(I_{h}\right)}}^{\text {min }}=I_{\min }$ in $A \otimes{ }^{\min } B$. Since $a \otimes b \in I_{h}, a \otimes b=$ $j_{\min }(a \otimes b) \in I_{\min }$ as well and, therefore, by Theorem 3.4, $a \otimes b \in I$.

Theorem 3.4 also allows us to deduce the following, which will be crucial in the proof of Theorem 3.13 .

Corollary 3.6. Let $A$ and $B$ be $C^{*}$-algebras and $I$ be a non-zero closed ideal in $A \otimes^{\gamma} B$. Then $I$ contains a non-zero elementary tensor and a product ideal.

Proof. From the Diagram 3.2 , we see that $I_{\min }$ is a non-zero closed ideal in $A \otimes^{\text {min }} B$. So, by [1, Proposition 4.5], $I_{\min }$ contains a non-zero elementary tensor, say, $a \otimes b$, and then, by Theorem 3.4, $a \otimes b \in I$. Also, if $J$ and $K$ are the closed ideals of $A$ and $B$, generated by $a$ and $b$, respectively, then the product ideal $J \otimes^{\gamma} K$ is contained in $I$.

This immediately yields the following analogue of [1, Corollary 4.7].

Corollary 3.7. Let $A$ and $B$ be $C^{*}$-algebras and $D$ be a Banach algebra. If $\pi: A \otimes^{\gamma} B \rightarrow D$ is a bounded homomorphism whose restriction to $A \otimes B$ is faithful, then so is $\pi$.

Proposition 3.8. Let $A$ and $B$ be $C^{*}$-algebras. Then a finite sum of closed product ideals in $A \otimes^{\gamma} B$ is closed.

Proof. It is enough to consider the sum of two product ideals. Let $J_{i}, K_{i}$ be closed ideals in $A$ and $B$, respectively, for $i=1,2$. By [7. Proposition 2.4], it is enough to show that $J_{1} \otimes^{\gamma} K_{1}$ has a bounded approximate identity. Since every closed ideal in a $C^{*}$-algebra possesses a bounded approximate identity, by [17, Lemma 3.1], $J_{1} \otimes^{\gamma} K_{1}$ possesses a bounded approximate identity.

From Theorem 2.6. Proposition 3.8 and the fact that a finite sum of closed ideals in a $C^{*}$ algebra is closed, we easily deduce the following.

Corollary 3.9. Let $\left\{J_{i}\right\}_{i=1}^{n}$ and $\left\{K_{j}\right\}_{j=1}^{m}$ be closed ideals in $C^{*}$-algebras $A$ and $B$, respectively. Then,

(1) $\left(\sum_{i} J_{i}\right) \otimes^{\gamma} B=\sum_{i} J_{i} \otimes^{\gamma} B$, and

(2) $A \otimes^{\gamma}\left(\sum_{j} K_{j}\right)=\sum_{j} A \otimes^{\gamma} K_{j}$.

Recall that a map $\pi: X \rightarrow Y$ between two Banach spaces is said to be a quotient map if it maps the open unit ball of $X$ onto that of $Y$. In particular, a quotient map is surjective. For two quotient maps $\varphi_{i}: X_{i} \rightarrow Y_{i}, \varphi_{1} \otimes \varphi_{2}$ extends to a quotient map $\varphi_{1} \otimes^{\gamma} \varphi_{2}: X_{1} \otimes^{\gamma} X_{2} \rightarrow$ $Y_{1} \otimes^{\gamma} Y_{2}$ - see [27, Proposition 2.5]. Anologous to [1, Theorem 2.4], 8, Proposition 7.1.7] and 21. Proposition 3.3], we obtain the following essential result:

Proposition 3.10. Let $X_{i}$ and $Y_{i}$ be Banach spaces and $\varphi_{i}: X_{i} \rightarrow Y_{i}, i=1,2$ be quotient maps. If $E_{1}$ and $E_{2}$ are closed subspaces of $Y_{1}$ and $Y_{2}$, respectively, then

$$
\left(\varphi_{1} \otimes^{\gamma} \varphi_{2}\right)^{-1}\left(\overline{E_{1} \otimes E_{2}}\right)=\overline{\operatorname{ker}\left(\varphi_{1}\right) \otimes X_{2}+\varphi_{1}^{-1}\left(E_{1}\right) \otimes \varphi_{2}^{-1}\left(E_{2}\right)+X_{1} \otimes \operatorname{ker}\left(\varphi_{2}\right)} .
$$

In particular, we have

$$
\operatorname{ker}\left(\varphi_{1} \otimes^{\gamma} \varphi_{2}\right)=\overline{\operatorname{ker}\left(\varphi_{1}\right) \otimes X_{2}+X_{1} \otimes \operatorname{ker}\left(\varphi_{2}\right)}
$$

Proof. Set $Z=\overline{\operatorname{ker}\left(\varphi_{1}\right) \otimes X_{2}+\varphi_{1}^{-1}\left(E_{1}\right) \otimes \varphi_{2}^{-1}\left(E_{2}\right)+X_{1} \otimes \operatorname{ker}\left(\varphi_{2}\right)}$. Recall that, for any subspace $W$ of a Banach space $X, W^{\perp \perp}=\bar{W}$, where $W^{\perp}:=\left\{\Phi \in X^{*}: \Phi(W)=(0)\right\}$ (Bipolar Theorem). So, it suffices to show that $\left(\left(\varphi_{1} \otimes \gamma \varphi_{2}\right)^{-1}\left(\overline{E_{1} \otimes E_{2}}\right)\right)^{\perp}=Z^{\perp}$.

Clearly, $\left(\left(\varphi_{1} \otimes^{\gamma} \varphi_{2}\right)^{-1}\left(\overline{E_{1} \otimes E_{2}}\right)\right)^{\perp} \subseteq Z^{\perp}$. For the reverse inclusion, let $f \in Z^{\perp}$. Since $\left(X_{1} \otimes^{\gamma} X_{2}\right)^{*}$ can be identified with the space of bounded bilinear forms on $X_{1} \times X_{2}([27, \S 2.2])$, 
there exists a bounded bilinear map $\tilde{f}: X_{1} \times X_{2} \rightarrow \mathbb{C}$ such that $f\left(a_{1} \otimes a_{2}\right)=\tilde{f}\left(a_{1}, a_{2}\right)$ for $a_{i} \in X_{i}$. Define $g: Y_{1} \times Y_{2} \rightarrow \mathbb{C}$ as $g\left(b_{1}, b_{2}\right)=\tilde{f}\left(a_{1}, a_{2}\right)$, where $\varphi_{i}\left(a_{i}\right)=b_{i}, i=1,2$. Since $\left.f\right|_{Z}=0, g$ is well defined, and it is also a bounded bilinear map. Thus $g$ can be identified with a unique element in $\left(Y_{1} \otimes^{\gamma} Y_{2}\right)^{*}$, say, $\tilde{g}$. It can be seen that $f=\tilde{g} \circ\left(\varphi_{1} \otimes^{\gamma} \varphi_{2}\right)$.

Now, take any $x$ in $\left(\varphi_{1} \otimes^{\gamma} \varphi_{2}\right)^{-1}\left(\overline{E_{1} \otimes E_{2}}\right)$. Then, by [27, Proposition 2.8], there exist bounded sequences $\left\{r_{n}\right\}$ and $\left\{s_{n}\right\}$ in $E_{1}$ and $E_{2}$, respectively, such that

$$
\left(\varphi_{1} \otimes \varphi_{2}\right)(x)=\sum_{n=1}^{\infty} r_{n} \otimes s_{n} .
$$

By surjectivity of $\varphi_{i}$ 's, for each $n \in \mathbb{N}$, fix $x_{n} \in \varphi_{1}^{-1}\left(r_{n}\right)$ and $y_{n} \in \varphi_{2}^{-1}\left(s_{n}\right)$. Then,

$$
f(x)=\tilde{g}\left(\sum_{n} r_{n} \otimes s_{n}\right)=\sum_{n} \tilde{g}\left(r_{n} \otimes s_{n}\right)=\sum_{n} f\left(x_{n} \otimes y_{n}\right)=0,
$$

which implies that $Z^{\perp} \subseteq\left(\left(\varphi_{1} \otimes^{\gamma} \varphi_{2}\right)^{-1}\left(\overline{E_{1} \otimes E_{2}}\right)\right)^{\perp}$.

We'll have instances ahead to appeal to the following useful consequence, wherein $i_{h}$ is as in Proposition 3.2 .

Corollary 3.11. Let $I$ and $J$ be closed ideals in $C^{*}$-algebras $A$ and $B$, respectively. Then, we have

$$
i_{h}\left(A \otimes^{\gamma} J+I \otimes^{\gamma} B\right)=\left(A \otimes^{h} J+I \otimes^{h} B\right) \cap i_{h}\left(A \otimes^{\gamma} B\right) .
$$

Proof. By Proposition 3.10, we have $\operatorname{ker}\left(\pi_{I} \otimes^{\gamma} \pi_{J}\right)=A \otimes^{\gamma} J+I \otimes^{\gamma} B$ and also, by 11, Corollary 2.6], $\operatorname{ker}\left(\pi_{I} \otimes^{h} \pi_{J}\right)=A \otimes^{h} J+I \otimes^{h} B$. Now, the diagram

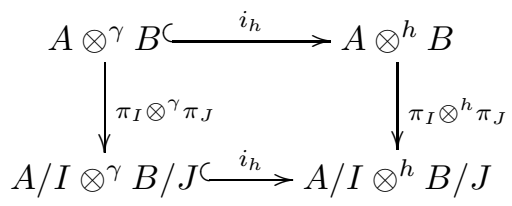

is easily seen to be commutative and we are done.

The following folklore result will be required in the proof of Theorem 3.13. (Note that, a part of it also follows from Proposition 3.10.)

Lemma 3.12. Let $A$ and $B$ be $C^{*}$-algebras, $J$ be a closed ideal in $B$ and $\pi: B \rightarrow B / J$ be the natural quotient map. Then, $\operatorname{ker}\left(\operatorname{Id} \otimes^{\gamma} \pi\right)=A \otimes^{\gamma} J$ and $\left(\operatorname{Id} \otimes^{\gamma} \pi\right)(Z)$ is closed for any closed subspace $Z$ in $A \otimes^{\gamma} B$. Additionally, if $Z$ contains $A \otimes^{\gamma} J$, then

$$
Z=\left(\operatorname{Id} \otimes^{\gamma} \pi\right)^{-1}\left(\left(\operatorname{Id} \otimes^{\gamma} \pi\right)(Z)\right) .
$$

Proof. Clearly, $A \otimes^{\gamma} J \subseteq \operatorname{ker}\left(\operatorname{Id} \otimes^{\gamma} \pi\right)$, where the inclusion is meaningful because of Theorem 2.6. Let $z \in \operatorname{ker}\left(\operatorname{Id} \otimes^{\gamma} \pi\right)$ and $\epsilon>0$. Then, by [27, Proposition 2.8], there exist bounded sequences $\left\{a_{n}\right\} \subset A$ and $\left\{b_{n}+J\right\} \subset B / J$ such that $\sum_{n} a_{n} \otimes\left(b_{n}+J\right)=0+J$ and $\sum_{n}\left\|a_{n}\right\|\left\|b_{n}+J\right\|<\epsilon$. Choose a sequence $\left\{x_{n}\right\} \subset J$ such that $\sum_{n}\left\|a_{n}\right\|\left\|b_{n}-x_{n}\right\|<\epsilon$. Then, it is easily seen that $\sum_{n} a_{n} \otimes x_{n}$ is absosutely convergent in $A \otimes^{\gamma} I$ and that $\left\|\sum_{n} a_{n} \otimes b_{n}-\sum_{n} a_{n} \otimes x_{n}\right\|_{\gamma}<\epsilon$, which implies that $A \otimes^{\gamma} I$ is dense in $\operatorname{ker}\left(\operatorname{Id} \otimes^{\gamma} \pi\right)$.

Finally, by [27, Proposition 2.3], $\left\|\operatorname{Id} \otimes^{\gamma} \pi\right\|=\|\operatorname{Id}\|\|\pi\|=1$ so that $\operatorname{Id} \otimes^{\gamma} \pi$ is a contraction and hence $\left(\operatorname{Id} \otimes^{\gamma} \pi\right)(Z)$ is closed. 
Note that Lemma 3.12 holds for Banach spaces and closed subspace as well. The same proof works in this generality.

With this, all ingredients are available to adapt the steps of [12, Theorem 3.1] to obtain a proof of the following ideal structure:

TheOREM 3.13. Let $A$ and $B$ be $C^{*}$-algebras where $A$ is topologically simple. Then every closed ideal of the Banach *-algebra $A \otimes^{\gamma} B$ is a product ideal of the form $A \otimes^{\gamma} J$ for some closed ideal $J$ in $B$. In particular, every closed ideal in $A \otimes^{\gamma} B$ is a *-ideal.

Proof. We will appeal to the usual Zorn's Lemma approach. Let $I$ be a non-zero closed ideal in $A \otimes^{\gamma} B$. Consider the collection

$$
\mathcal{F}:=\left\{J \subseteq B: J \text { is a closed ideal in } B \text { and } A \otimes^{\gamma} J \subseteq I\right\},
$$

where the inclusion $A \otimes^{\gamma} J \subseteq I$ makes sense by Theorem 2.6. By Corollary 3.6, $I$ contains a non-zero elementary tensor, say, $a \otimes b$. If $K$ and $J$ are the non-zero closed ideals in $A$ and $B$ generated by $a$ and $b$, respectively, then by simplicity of $A$, we have $K=A$ and $A \otimes^{\gamma} J \subseteq I$. In particular, $\mathcal{F} \neq \emptyset$.

We saw in Corollary 3.9 that $A \otimes^{\gamma}\left(\sum_{i} J_{i}\right)=\sum_{i}\left(A \otimes^{\gamma} J_{i}\right)$ for any finite collection of closed ideals $\left\{J_{i}\right\}$ in $B$. So, with respect to the partial order given by set inclusion, every chain $\left\{J_{i}: i \in \Lambda\right\}$ in $\mathcal{F}$ has an upper bound in $\mathcal{F}$, namely, the closure of the ideal $\left\{\sum_{\text {finite }} x_{i}: x_{i} \in J_{i}\right\}$, implying thereby that there exists a maximal element, say $J$, in $\mathcal{F}$.

The obvious thing to do now is to try to show that $A \otimes^{\gamma} J=I$. Consider the canonical map Id $\otimes^{\gamma} \pi: A \otimes^{\gamma} B \rightarrow A \otimes^{\gamma}(B / J)$. By Lemma 3.12, we have $\operatorname{ker}\left(\operatorname{Id} \otimes^{\gamma} \pi\right)=A \otimes^{\gamma} J$ and that $\widetilde{I}:=\left(\operatorname{Id} \otimes^{\gamma} \pi\right)(I)$ is a closed ideal in $A \otimes^{\gamma}(B / J)$. It now suffices to show that $\widetilde{I}=(0)$. If $\widetilde{I} \neq(0)$, then, again by Corollary $3.6, \widetilde{I}$ contains a non-zero elementary tensor, say, $a \otimes(b+J)$. Observe that $b \notin J$ and

$$
a \otimes b \in\left(\operatorname{Id} \otimes^{\gamma} \pi\right)^{-1}(a \otimes(b+J)) \in\left(\operatorname{Id} \otimes^{\gamma} \pi\right)^{-1}\left(\left(\operatorname{Id} \otimes^{\gamma} \pi\right)(I)\right)=I
$$

by Lemma 3.12. Let $K$ denote the closed ideal in $B$ generated by $b$ and $J$. Note that $J \subsetneq K$. Since $A$ is simple, it equals the closed ideal generated by $a$ and we obtain $A \otimes^{\gamma} K \subseteq I$, i.e., $K \in \mathcal{F}$ contradicting the maximality of $J$ in $\mathcal{F}$.

In view of Theorems 2.6, 3.13 and Lemma 3.12, we obtain the following analogue of [28, Corollary 4.21], [1, Theorem 5.1] and of [17, Theorem 3.7].

Corollary 3.14. Let $A$ and $B$ be $C^{*}$-algebras. Then the Banach *-algebra $A \otimes{ }^{\gamma} B$ is topologically simple if and only if $A$ and $B$ are both topologically simple.

If $A$ contains only finitely many closed ideals and $\alpha$ is either the Haagerup or the operator space projective tensor product, then every closed ideal in the Banach algebra $A \otimes^{\alpha} B$ is a finite sum of product ideals, - see [1, 21]. We now make another use of Theorem 3.13 to prove its analogue for $A \otimes^{\gamma} B$. We'll use the following useful observation made in the proof of [1, Theorem $5.3]$.

LEMmA 3.15. Let $A$ and $B$ be $C^{*}$-algebras and $I$ be a simple closed ideal in $A$. If $K$ is a closed ideal in the Banach algebra $A \otimes^{h} B$, then $K \cap\left(I \otimes^{h} B\right)=I \otimes^{h} J$ for some closed ideal $J$ in $B$, and,

$$
K \subseteq A \otimes^{h} J+M \otimes^{h} B,
$$

where $M$ is the closed ideal ann $(I):=\{x \in A: x I=I x=(0)\}$.

TheOrem 3.16. Let $A$ and $B$ be $C^{*}$-algebras and suppose $A$ contains only finitely many closed ideals. Then every closed ideal in the Banach *-algebra $A \otimes^{\gamma} B$ is a finite sum of product ideals. 
Proof. The proof is given by induction on the number of closed ideals in $A$, call it $\nu(A)$. If $\nu(A)=2$, then we are done by Theorem 3.13. Let $n>2$ and suppose that the assertion holds for all $C^{*}$-algebras with less than $n$ closed ideals, and let $A$ be a $C^{*}$-algebra with $\nu(A)=n$.

Pick a minimal non-zero closed ideal, say $I$, in $A$, which is clearly simple. Let $K$ be a closed ideal in $A \otimes^{\gamma} B$, then $I \otimes^{\gamma} B \subseteq A \otimes^{\gamma} B$ by Theorem 2.6] so that $K \cap\left(I \otimes^{\gamma} B\right)$ is a closed ideal in $I \otimes^{\gamma} B$. By Theorem 3.13 it is then equal to $I \otimes^{\gamma} J$ for some closed ideal $J$ in $B$.

Consider the closed ideal $K_{h}:={\overline{i_{h}(K)}}^{h}$ in the Banach algebra $A \otimes^{h} B$ (where $i_{h}$ is as in Proposition 3.2). By Lemma 3.15, $K_{h} \cap\left(I \otimes^{h} B\right)=I \otimes^{h} J_{1}$ and $K_{h} \subseteq A \otimes^{h} J_{1}+M \otimes^{h} B$ for some closed ideal $J_{1}$ in $B$, where $M=\operatorname{ann}(I)$.

We wish to show, in fact, that $K \subseteq A \otimes^{\gamma} J+M \otimes^{\gamma} B$ as well. Note that $K \subseteq i_{h}^{-1}\left(K_{h}\right) \subseteq$ $i_{h}^{-1}\left(A \otimes^{h} J_{1}+M \otimes^{h} B\right)$ and, by Corollary 3.11, $i_{h}^{-1}\left(A \otimes^{h} J_{1}+M \otimes^{h} B\right)=A \otimes^{\gamma} J_{1}+M \otimes^{\gamma} B$. So, it suffices to show that $J_{1} \subset J$. Note that, if $y \in J_{1}$, then $x \otimes y \in I \otimes^{h} J_{1} \subset K_{h}$ for any fixed $0 \neq x \in I$, so that, by Corollary 3.5, $x \otimes y \in K$ implying further that $x \otimes y \in K \cap\left(I \otimes^{\gamma} B\right)=I \otimes^{\gamma} J$. Choose a $\varphi \in A^{*}$ such that $\varphi(x) \neq 0$, then $R_{\varphi}(x \otimes y)=\varphi(x) y \in J$, where $R_{\varphi}: A \otimes B \rightarrow B$ is the right slice map given by $R_{\varphi}\left(\sum_{1}^{n} a_{i} \otimes b_{i}\right)=\sum_{1}^{n} \varphi\left(a_{i}\right) b_{i}$. Hence $y \in J$.

We now claim that $K \cap\left(A \otimes^{\gamma} J+M \otimes^{\gamma} B\right)=K \cap\left(A \otimes^{\gamma} J\right)+K \cap\left(M \otimes^{\gamma} B\right)$ and show that each of the two closed ideals appearing in the sum on the right hand side, by induction hypothesis, are finite sums of closed ideals, which will then complete the proof.

We first prove that $L:=K \cap\left(A \otimes^{\gamma} J\right)$ is a finite sum of closed ideals. Clearly $L$ contains $I \otimes^{\gamma} J$.

Corresponding to the complete quotient map $\pi_{I}: A \rightarrow A / I$, we have a quotient map $\pi_{I} \otimes^{\gamma} I d$ : $A \otimes^{\gamma} J \rightarrow A / I \otimes^{\gamma} J$. By Lemma 3.12 $\operatorname{ker}\left(\pi_{I} \otimes^{\gamma} I d\right)=I \otimes^{\gamma} J$ and $\left(\pi_{I} \otimes^{\gamma} I d\right)(L)$ is a closed ideal in $A / I \otimes^{\gamma} J$. Since $\nu(A / I) \leq n-1$, by induction hypothesis, $\left(\pi_{I} \otimes^{\gamma} I d\right)(L)=\sum_{r=1}^{k} I_{r} \otimes^{\gamma} J_{r}$, where $I_{r}$ and $J_{r}$ are closed ideals in $A / I$ and $J$, respectively. Thus, by Lemma 3.12 and Proposition 3.10, $L=\sum_{r=1}^{k} \pi_{I}^{-1}\left(I_{r}\right) \otimes^{\gamma} J_{r}+I \otimes^{\gamma} J$.

Further, since $M$ cannot contain $I$, we have $\nu(M) \leq n-1$. So, by induction hypothesis, the closed ideal $K \cap\left(M \otimes^{\gamma} B\right)$ is a finite sum of product ideals.

Finally, it is easy to see that $K \cap\left(A \otimes^{\gamma} J\right)+K \cap\left(M \otimes^{\gamma} B\right) \subseteq K \cap\left(A \otimes^{\gamma} J+M \otimes^{\gamma} B\right)$. Let $z \in K \cap\left(A \otimes^{\gamma} J+M \otimes^{\gamma} B\right)$. By [12, Proposition 4.11], the closed ideal $A \otimes^{\gamma} J+M \otimes^{\gamma} B$ possesses a quasi-central approximate identity, say $\left\{e_{\lambda}\right\}$, which as in [1, Lemma 3.3], can be taken to be of the form $e_{\lambda}=f_{\lambda}+g_{\lambda}-f_{\lambda} g_{\lambda}$ for some quasi-central approximate identities $\left\{f_{\lambda}\right\}$ and $\left\{g_{\lambda}\right\}$ in $A \otimes^{\gamma} J$ and $M \otimes^{\gamma} B$, respectively. Then, $e_{\lambda} z \rightarrow z$ and $z e_{\lambda} \in K \cap\left(A \otimes^{\gamma} J\right)+K \cap\left(M \otimes^{\gamma} B\right)$ for every $\lambda$. This implies that $K \cap\left(A \otimes^{\gamma} J\right)+K \cap\left(M \otimes^{\gamma} B\right)$ is dense in $K \cap\left(A \otimes^{\gamma} J+M \otimes^{\gamma} B\right)$ and, by Proposition 3.8, we know that $K \cap\left(A \otimes^{\gamma} J\right)+K \cap\left(M \otimes^{\gamma} B\right)$, being a finite sum of product ideals, is closed.

Based on above discussion, we easily deduce the following:

Corollary 3.17. Let $A$ and $B$ be $C^{*}$-algebras and suppose $A$ contains only finitely many closed ideals. Then, the following hold:

(1) A finite sum of closed ideals in $A \otimes^{\gamma} B$ is also a closed ideal.

(2) Every closed ideal of $A \otimes^{\gamma} B$ contains a bounded approximate unit.

(3) Every closed ideal of $A \otimes^{\gamma} B$ is $*$-closed.

Some Examples. We can now reap some immediate fruits of Theorem 3.16 and Corollary 3.17

(1) For any separable Hilbert space $H$, analogous to the structure of closed ideals of $B(H) \otimes^{\alpha}$ $B(H)$ for $\otimes^{\alpha}=\otimes^{h}$ and $\widehat{\otimes}$ (see [1, 16]), $B(H) \otimes^{\gamma} B(H)$ contains only four non-trivial closed ideals, namely, $B(H) \otimes^{\gamma} K(H)+K(H) \otimes^{\gamma} B(H), B(H) \otimes^{\gamma} K(H), K(H) \otimes^{\gamma} B(H)$ and $K(H) \otimes^{\gamma} K(H)$. In particular, $B(H) \otimes^{\gamma} B(H)$ has a unique maximal ideal, namely, $B(H) \otimes^{\gamma} K(H)+K(H) \otimes^{\gamma} B(H)$. 
(2) For a locally compact Hausdorff space $X$ and a separable Hilbert space $H$, the closed ideals of $B(H) \otimes^{\gamma} C_{0}(X)$ are given by $\sum_{i=1}^{n} I_{i} \otimes^{\gamma} I\left(E_{i}\right)$, for closed subsets $E_{i}$ of $X$, where $I_{i}=K(H)$ or $B(H)$, and

$$
I\left(E_{i}\right):=\left\{f \in C_{0}(X): f(x)=0 \text { for all } x \in E_{i}\right\} .
$$

Note that, by Theorem 3.21, $B(H) \otimes^{\gamma} I(\{x\})+K(H) \otimes^{\gamma} C_{0}(X)$ is a maximal ideal in $B(H) \otimes{ }^{\gamma} C_{0}(X)$ for each $x \in C_{0}(X)$.

3.1. Minimal and maximal ideals of $A \otimes^{\gamma} B$. The structure of closed minimal ideals of $A \otimes^{\gamma} B$ turns out to be an immediate consequence of Corollary 3.6.

Proposition 3.18. Let $A$ and $B$ be $C^{*}$-algebras. Then, a closed ideal $J$ in $A \otimes^{\gamma} B$ is minimal if and only if it is a product ideal of the form $J=K \otimes^{\gamma} L$ for some minimal closed ideals $K$ and $L$ in $A$ and $B$, respectively.

The proof of [17, Proposition 3.9] works verbatim for above identification.

In order to analyze the structure of maximal ideals, we will use the concept of Wiener property for Banach $*$-algebras. Recall that a Banach $*$-algebra $A$ said to have the Wiener property if every proper closed ideal of $A$ is annihilated by some irreducible $*$-representation of $A$ on some Hilbert space. It is well known that every $C^{*}$-algebra has Wiener property.

Lemma 3.19. Let $A$ and $B$ be $C^{*}$-algebras. Then, the Banach *-algebra $A \otimes^{\gamma} B$ has the Wiener property.

Proof. Consider a proper closed two-sided ideal $J$ of $A \otimes^{\gamma} B$. By Theorem 3.4. $J_{\min }$ is also a proper closed two-sided ideal of the $C^{*}$-algebra $A \otimes{ }^{\min } B$. Since every $C^{*}$-algebra has the Wiener property, $J_{\min }$ is annihilated by an irreducible $*$-representation, say, $\pi: A \otimes^{\min } B \rightarrow$ $B(H)$. So, we have a $*$-representation $\hat{\pi}:=\pi \circ i$ of $A \otimes^{\gamma} B$ on $H$ with $\hat{\pi}(J)=\{0\}$, where $i: A \otimes^{\gamma} B \rightarrow A \otimes^{\min } B$ is the canonical injective $*$-homomorphism as in Proposition 3.2. Also, the relation $\hat{\pi}(A \otimes B)=\pi(A \otimes B)$ yields

$$
\hat{\pi}\left(A \otimes^{\gamma} B\right)^{\prime} \subseteq \hat{\pi}(A \otimes B)^{\prime}=\pi(A \otimes B)^{\prime}=\pi\left(A \otimes^{\min } B\right)^{\prime}=\mathbb{C} I .
$$

Thus, $\hat{\pi}$ is irreducible and $A \otimes^{\gamma} B$ has Wiener property.

Lemma 3.20. Let $A$ and $B$ be $C^{*}$-algebras and $\pi$ be an irreducible *-representation of $A \otimes^{\gamma} B$ on a Hilbert space $H$. Then there exist $*$-representations $\pi_{1}$ and $\pi_{2}$ of $A$ and $B$, respectively, on $H$ with commuting ranges such that

$$
\pi(a \otimes b)=\pi_{1}(a) \pi_{2}(b) \text { for all } a \in A, b \in B .
$$

Moreover, $\pi_{1}$ and $\pi_{2}$ are both factor representations.

Proof. Since $\pi$ is a $*$-representation of $A \otimes B$, by [28, Lemma IV.4.1], there exist $*$-representations $\pi_{1}$ and $\pi_{2}$ of $A$ and $B$ on $H$ with commuting ranges such that

$$
\pi(a \otimes b)=\pi_{1}(a) \pi_{2}(b) \text { for all } a \in A, b \in B .
$$

Now, $\pi(A \otimes B)=\pi_{1}(A) \pi_{2}(B)$, so that $\pi\left(A \otimes^{\gamma} B\right) \subseteq \overline{\pi_{1}(A) \pi_{2}(B)}$. Irreducibility of $\pi$ gives

$$
\left(\pi_{1}(A) \pi_{2}(B)\right)^{\prime}=\left(\overline{\pi_{1}(A) \pi_{2}(B)}\right)^{\prime} \subseteq \pi\left(A \otimes^{\gamma} B\right)^{\prime}=\mathbb{C} I .
$$


If $M:=\pi_{1}(A)^{\prime \prime}$, then we have

$$
\begin{aligned}
M \cap M^{\prime} & =\pi_{1}(A)^{\prime \prime} \cap \pi_{1}(A)^{\prime} \\
& =\left(\pi_{1}(A)^{\prime} \cup \pi_{1}(A)\right)^{\prime} \\
& \subseteq\left(\pi_{2}(B) \cup \pi_{1}(A)\right)^{\prime} \quad\left(\text { as } \pi_{2}(B) \subseteq \pi_{1}(A)^{\prime}\right) \\
& =\pi_{1}(A)^{\prime} \cap \pi_{2}(B)^{\prime} \\
& \subseteq\left\{\pi_{1}(A) \pi_{2}(B)\right\}^{\prime} \\
& =\mathbb{C} I .
\end{aligned}
$$

Thus, $\pi_{1}$ (and similarly $\pi_{2}$ ) is a factor representation.

Analogous to [1, Theorem 5.6], [17, Theorem 3.10] and [16, Theorem 9], we now obtain the following characterizations of maximal and maximal modular ideals.

Theorem 3.21. Let $A$ and $B$ be $C^{*}$-algebras. Then, a closed ideal $J$ in $A \otimes^{\gamma} B$ is maximal if and only if it is of the form $J=A \otimes^{\gamma} N+M \otimes^{\gamma} B$ for some maximal ideals $M$ and $N$ in $A$ and $B$, respectively.

Proof. Let $J=A \otimes^{\gamma} N+M \otimes^{\gamma} B$, where $M$ and $N$ are maximal ideals in $A$ and $B$, respectively. Note that, by Proposition 3.8, $A \otimes^{\gamma} N+M \otimes^{\gamma} B$ is a closed ideal in $A \otimes^{\gamma} B$. For the canonical quotient maps $\pi_{1}: A \rightarrow A / M$ and $\pi_{2}: B \rightarrow B / N$, we have $J=\operatorname{ker}\left(\pi_{1} \otimes \pi_{2}\right)$ and there is an isomorphism between $\left(A \otimes^{\gamma} B\right) / J$ and $(A / M) \otimes^{\gamma}(B / N)$ by Proposition 3.10. Since $A / M$ and $B / N$ are both simple, so is $\left(A \otimes^{\gamma} B\right) / J$, by Corollary 3.14. Thus, $J$ is maximal in $A \otimes^{\gamma} B$.

Conversely, let $J$ be a maximal ideal in $A \otimes^{\gamma} B$. As seen in Lemma 3.19, $A \otimes^{\gamma} B$ has the Wiener property; so, there exists a non-zero irreducible $*$-representation $\pi$ of $A \otimes^{\gamma} B$ on a Hilbert space $H$, such that $\pi(J)=(0)$. Then, by Lemma 3.20, there exist factor $*$-representations $\pi_{1}$ and $\pi_{2}$ of $A$ and $B$ on $H$ with commuting ranges such that $\pi(a \otimes b)=\pi_{1}(a) \pi_{2}(b)$ for all $a \in A$ and $b \in B$. Set $M=\operatorname{ker} \pi_{1}, N=\operatorname{ker} \pi_{2}$ and $L=A \otimes^{\gamma} N+M \otimes^{\gamma} B$. We first show that $L=J$.

Clearly, $\pi\left(M \otimes^{\gamma} B\right)=(0)=\pi\left(A \otimes^{\gamma} N\right)$, which gives $\pi(J+L)=(0)$. Since $\pi$ is non-zero, this shows that $J+L$ is a proper ideal of $A \otimes^{\gamma} B$. Since $J+L$ contains $J$, by maximality of $J$, we have $L \subseteq J$, i.e., $A \otimes^{\gamma} N+M \otimes^{\gamma} B \subseteq J$.

By Proposition 3.10 and Proposition 3.8 we have $\operatorname{ker}\left(\pi_{M} \otimes^{\gamma} \pi_{N}\right)=A \otimes^{\gamma} N+M \otimes^{\gamma} B$, so, for the reverse inclusion, it suffices to show that $J \subseteq \operatorname{ker}\left(\pi_{M} \otimes^{\gamma} \pi_{N}\right)$, where $\pi_{M}$ and $\pi_{N}$ are the natural quotient maps. Note that the representations $\pi_{1}$ and $\pi_{2}$ induce faithful commuting representations $\tilde{\pi}_{1}$ of $A / M$ and $\tilde{\pi}_{2}$ of $B / N$ on $H$. Then, by a universal property of $\otimes$ max (see [28, Proposition IV.4.7]), there exists a bounded $*$-representation $\pi_{0}:(A / M) \otimes{ }^{\max }(B / N) \rightarrow B(H)$ such that $\pi_{0}(x \otimes y)=\tilde{\pi}_{1}(x) \tilde{\pi}_{2}(y)$ for all $x \in A / M$ and $y \in B / N$. Since $\|\cdot\|_{\max } \leq\|\cdot\|_{\gamma}$, the identity map on $(A / M) \otimes(B / N)$ extends to a contractive $*$-homomorphism, say, $i:(A / M) \otimes \gamma(B / N) \rightarrow$ $(A / M) \otimes^{\max }(B / N)$. In particular, $\theta:=\pi_{0} \circ i$ is a $*$-representation of $(A / M) \otimes^{\gamma}(B / N)$ on $H$.

It is easy to verify that $\pi=\theta \circ\left(\pi_{M} \otimes^{\gamma} \pi_{N}\right)$ on $A \otimes B$, so by continuity we have $\pi=$ $\theta \circ\left(\pi_{M} \otimes^{\gamma} \pi_{N}\right)$, which further gives $\theta\left(\left(\pi_{M} \otimes^{\gamma} \pi_{N}\right)(J)\right)=0$. We now claim that $\theta$ is faithful on $(A / M) \otimes^{\gamma}(B / N)$, which will yield $\left(\pi_{M} \otimes^{\gamma} \pi_{N}\right)(J)=(0)$, as was asserted above. Note that, by Corollary 3.7 it suffices to show that $\theta$ is faithful on $(A / M) \otimes(B / N)$. Since $\pi_{1}$ and $\pi_{2}$ are both factor representations, so are the representations $\tilde{\pi}_{1}$ and $\tilde{\pi}_{2}$ because

$$
\tilde{\pi}_{1}(A / M)^{\prime \prime}=\pi_{1}(A)^{\prime \prime} \quad \text { and } \quad \tilde{\pi}_{2}(B / N)^{\prime \prime}=\pi_{2}(B)^{\prime \prime} .
$$

Now, for the factor $\mathcal{R}=\tilde{\pi}_{1}(A / M)^{\prime \prime}$, the map

$$
\mathcal{R} \otimes \mathcal{R}^{\prime} \ni \Sigma_{i=1}^{n} x_{i} \otimes x_{i}^{\prime} \stackrel{\rho}{\mapsto} \Sigma_{i=1}^{n} x_{i} x_{i}^{\prime} \in B(H)
$$


is an injective homomorphism, by [28, Proposition IV.4.20]. Suppose $\theta\left(\sum_{i=1}^{n} x_{i} \otimes y_{i}\right)=0$ for some $\sum_{i=1}^{n} x_{i} \otimes y_{i} \in(A / M) \otimes(B / N)$, which gives

$$
0=\theta\left(\sum_{i} x_{i} \otimes y_{i}\right)=\sum_{i} \tilde{\pi}_{1}\left(x_{i}\right) \tilde{\pi}_{2}\left(y_{i}\right)=\rho\left(\sum_{i} \tilde{\pi}_{1}\left(x_{i}\right) \otimes \tilde{\pi}_{2}\left(y_{i}\right)\right) .
$$

Note that $\tilde{\pi}_{2}\left(y_{i}\right) \in \tilde{\pi}_{1}(A / M)^{\prime}=\mathcal{R}^{\prime \prime \prime}=\mathcal{R}^{\prime}$ for all $i$. Since $\rho$ is injective, we obtain

$$
\left(\tilde{\pi}_{1} \otimes \tilde{\pi}_{2}\right)\left(\sum_{i} x_{i} \otimes y_{i}\right)=\sum_{i} \tilde{\pi}_{1}\left(x_{i}\right) \otimes \tilde{\pi}_{2}\left(y_{i}\right)=0 .
$$

Further, since $\tilde{\pi}_{1}$ and $\tilde{\pi}_{2}$ are both injective, so is $\tilde{\pi}_{1} \otimes \tilde{\pi}_{2}$ and hence $\sum_{i} x_{i} \otimes y_{i}=0$. This proves our claim.

Finally, since $J=\operatorname{ker}\left(\pi_{M} \otimes^{\gamma} \pi_{N}\right),\left(A \otimes^{\gamma} B\right) / J$ is isomorphic to $(A / M) \otimes^{\gamma}(B / N)$. So, by Corollary 3.14, it follows that $M$ and $N$ are both maximal in $A$ and $B$, respectively.

It is known that an ideal in a Banach algebra is maximal modular if and only if it is maximal and modular. The above structure of maximal ideals immediately yields the structure of maximal modular ideals as well.

Theorem 3.22. Let $A$ and $B$ be $C^{*}$-algebras. Then, a closed ideal $J$ of $A \otimes^{\gamma} B$ is maximal modular if and only if it is of the form $J=A \otimes^{\gamma} N+M \otimes^{\gamma} B$ for some maximal modular ideals $M$ and $N$ in $A$ and $B$, respectively.

Proof. Let $J=A \otimes^{\gamma} N+M \otimes^{\gamma} B$ for some maximal modular ideals $M$ and $N$ in $A$ and $B$, respectively. Since $M$ and $N$ are both maximal ideals, so is $J$, by Theorem 3.21. Also, by Proposition 3.10, $\left(A \otimes^{\gamma} B\right) / J$ and $(A / M) \otimes^{\gamma}(B / N)$ are isomorphic Banach $*$-algebras. Since $A / M$ and $B / N$ are both unital, so is $\left(A \otimes^{\gamma} B\right) / J$. In particular, $J$ is modular.

Conversely, suppose $J$ is a maximal modular ideal in $A \otimes^{\gamma} B$. Again, by Theorem 3.21 $J$, being maximal, is of the form $J=A \otimes^{\gamma} M+I \otimes^{\gamma} N$ for some maximal ideals $M$ and $N$ in $A$ and $B$, respectively. As seen in previous paragraph, $\left(A \otimes^{\gamma} B\right) / J$ is isomorphic to $(A / M) \otimes^{\gamma}(B / N)$; in particular, the latter space is unital. Therefore, by [25. Theorem 1], $A / M$ and $B / N$ are both unital; so that $M$ and $N$ are both modular as well.

\section{Hull-KeRnel TOPOLOGY}

As in Introduction, for any Banach algebra $A, I d(A)$ (resp., $\left.I d^{\prime}(A)\right)$ denotes the set of closed ideals (resp., proper closed ideals) of $A$. And, for any algebra $A, \mathcal{M}(A)$ (resp., $\mathcal{M}_{m}(A)$ ) denotes the set of maximal (resp., maximal modular) ideals of $A$.

Before discussing hull-kernel topology, we briefly outline another topology on $\operatorname{Id}(A)$ for a Banach algebra $A$, which agrees with hull-kernel topology on the set of maximal ideals and is called the $\tau_{w}$-topology $([3, \S 2])$. A subbasis for $\tau_{w}$-topology is given by the collection

$$
\{U(J):=\{I \in I d(A): I \nsupseteq J\}, J \in I d(A) \cup\{\emptyset\}\},
$$

where $U(\emptyset):=A$. Note that $U(A)=I d^{\prime}(A), U((0))=\emptyset$ and $U(\emptyset)$ is the only subbasic set that contains $A$. $\operatorname{Id}(A)$ is a $T_{0}$ space with respect to $\tau_{w}$-topology (3).

For $C^{*}$-algebras $A$ and $B$, consider the map $\Phi: \operatorname{Id}(A) \times \operatorname{Id}(B) \rightarrow \operatorname{Id}\left(A \otimes^{\gamma} B\right)$ defined by

$$
\Phi(I, J)=\operatorname{ker}\left(\pi_{I} \otimes^{\gamma} \pi_{J}\right)=A \otimes^{\gamma} J+I \otimes^{\gamma} B,
$$

where $\pi_{I}: A \rightarrow A / I$ and $\pi_{J}: B \rightarrow B / J$ are the canonical quotient maps. Note that the last equality in (4.1) follows from Proposition 3.10 and Proposition 3.8 Also, If $(I, J) \in I^{\prime}(A) \times$ $I d^{\prime}(B)$, then $\pi_{I} \otimes^{\gamma} \pi_{J} \neq 0$ so that $\operatorname{ker}\left(\pi_{I} \otimes^{\gamma} \pi_{J}\right)$ is proper. Hence $\Phi$ maps $I d^{\prime}(A) \times I d^{\prime}(B)$ into $I d^{\prime}\left(A \otimes^{\gamma} B\right)$. Analogous to [4, Lemma 1.4] and [24, Lemma 2.5], we obtain the following: 
Lemma 4.1. Let $A$ and $B$ be $C^{*}$-algebras. Then, $\Phi: \operatorname{Id}(A) \times \operatorname{Id}(B) \rightarrow \operatorname{Id}\left(A \otimes^{\gamma} B\right)$ is $\tau_{w^{-}}$ continuous.

Proof. Consider the diagram

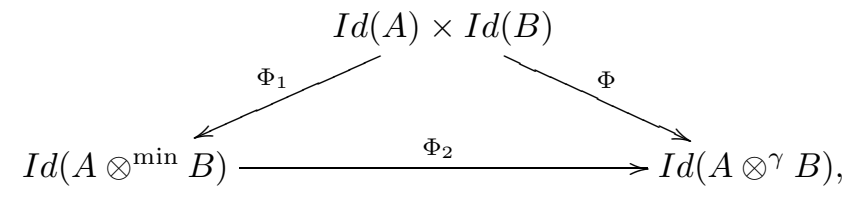

where $\Phi_{1}(I, J):=\operatorname{ker}\left(\pi_{I} \otimes^{\min } \pi_{J}\right)$ and $\Phi_{2}(K):=i^{-1}(K), i$ being the injective contractive *-homomorphism from $A \otimes^{\gamma} B \rightarrow A \otimes^{\min } B$ (as in Proposition [3.2). It is known that $\Phi_{1}$ is $\tau_{w}$-continuous - see [24, Lemma 2.5]. So, it suffices to show that this diagram commutes and that $\Phi_{2}$ is $\tau_{w}$-continuous.

In order to establish commutativity of the diagram, we just need to verify that

$$
\operatorname{ker}\left(\pi_{I} \otimes^{\gamma} \pi_{J}\right)=i^{-1}\left(\operatorname{ker}\left(\pi_{I} \otimes^{\min } \pi_{J}\right)\right) .
$$

For $z \in A \otimes^{\gamma} B$, let $\left\{z_{n}\right\}$ be a sequence in $A \otimes B$ such that $\left\|z_{n}-z\right\|_{\gamma} \rightarrow 0$. Let $\hat{i}:(A / I) \otimes^{\gamma}$ $(B / J) \rightarrow(A / I) \otimes^{\min }(B / J)$ be the injective continuous homomorphism. Then the sequence $\left\{\hat{i}\left(\left(\pi_{I} \otimes^{\gamma} \pi_{J}\right)\left(z_{n}\right)\right)=\left(\pi_{I} \otimes^{\gamma} \pi_{J}\right)\left(z_{n}\right)\right\}$ converges to $\hat{i}\left(\left(\pi_{I} \otimes^{\gamma} \pi_{J}\right)(z)\right)$ in $(A / I) \otimes^{\min }(B / J)$. Since $\|\cdot\|_{\min } \leq\|\cdot\|_{\gamma}$ and $i$ is identity on $A \otimes B,\left\|z_{n}-i(z)\right\|_{\min } \rightarrow 0$ as well. So, $\left(\pi_{I} \otimes{ }^{\min }\right.$ $\left.\pi_{J}\right)\left(z_{n}\right) \longrightarrow\left(\pi_{I} \otimes^{\min } \pi_{J}\right)(i(z))$ in $(A / I) \otimes^{\min }(B / J)$. Since both the mappings $\pi_{I} \otimes^{\gamma} \pi_{J}$ and $\pi_{I} \otimes{ }^{\min } \pi_{J}$ agree on $A \otimes B$, by continuity, we have

$$
\hat{i}\left(\left(\pi_{I} \otimes^{\gamma} \pi_{J}\right)(z)\right)=\left(\pi_{I} \otimes^{\min } \pi_{J}\right)(i(z)) .
$$

The required relationship now follows from injectivity of $\hat{i}$.

Next, we show $\Phi_{2}$ is $\tau_{w}$-continuous. For a subbasic open set $U(K)$ of $\operatorname{Id}\left(A \otimes^{\gamma} B\right)$ for some $K \in I d\left(A \otimes^{\gamma} B\right)$, we have $\Phi_{2}^{-1}(U(K))=U\left(K_{\text {min }}\right)$. Indeed, for $P \in I d\left(A \otimes^{\min } B\right)$,

$$
\begin{aligned}
P \in \Phi_{2}^{-1}(U(K)) & \Longleftrightarrow i^{-1}(P) \in U(K) \\
& \Longleftrightarrow i^{-1}(P) \nsupseteq K \\
& \Longleftrightarrow P \nsupseteq K_{\min } \quad(:=\overline{i(K)}) \\
& \Longleftrightarrow P \in U\left(K_{\min }\right) .
\end{aligned}
$$

Thus, $\Phi_{2}$ is $\tau_{w}$-continuous.

Lemma 4.2. Let $A$ and $B$ be $C^{*}$-algebras and $\left(I_{i}, J_{i}\right) \in I d^{\prime}(A) \times I d^{\prime}(B), i=1,2$ be such that $A \otimes^{\gamma} J_{1}+I_{1} \otimes^{\gamma} B \subseteq A \otimes^{\gamma} J_{2}+I_{2} \otimes^{\gamma} B$. Then $I_{1} \subseteq I_{2}$ and $J_{1} \subseteq J_{2}$.

Proof. For a fixed $a \in I_{1}$ and any $b \in B$ we have $a \otimes b \in \operatorname{ker}\left(\pi_{I_{1}} \otimes^{\gamma} \pi_{J_{1}}\right) \subseteq \operatorname{ker}\left(\pi_{I_{2}} \otimes^{\gamma} \pi_{J_{2}}\right)$, by the given condition. This yields $\pi_{I_{2}}(a) \otimes \pi_{J_{2}}(b)=0$ for every $b \in B$. Since $J_{2}$ is proper, $\pi_{J_{2}}(b) \neq 0$ for some $b \in B$. So, we must have $\pi_{I_{2}}(a)=0$, that is, $a \in I_{2}$. Similarly, we obtain $J_{1} \subseteq J_{2}$.

We now obtain the following analogue of [4, Theorem 1.5], [24, Theorem 2.6] and [18, Proposition $1.1(\mathrm{v})]$.

Theorem 4.3. Let $A$ and $B$ be $C^{*}$-algebras. Then, $\Phi$ maps $I d^{\prime}(A) \times I d^{\prime}(B)$ homeomorphically onto its image which is dense in $I d^{\prime}\left(A \otimes^{\gamma} B\right)$.

Proof. For a closed ideal $K$ in $A \otimes^{\gamma} B$, define

$$
K_{A}=\{a \in A: a \otimes B \subseteq K\} \quad \text { and } \quad K_{B}=\{b \in B: A \otimes b \subseteq K\} .
$$


By an easy application of continuous functional calculus, it is immediately seen that $K_{A}$ and $K_{B}$ are closed ideals in $A$ and $B$, respectively. Define $\Psi: \operatorname{Id}\left(A \otimes^{\gamma} B\right) \rightarrow \operatorname{Id}(A) \times \operatorname{Id}(B)$ by $\Psi(K)=\left(K_{A}, K_{B}\right)$. Then $\Psi \circ \Phi$ equals identity on $I d^{\prime}(A) \times I d^{\prime}(B)$. To see this, consider $(I, J) \in I d^{\prime}(A) \times I d^{\prime}(B)$ and set $K=\Phi(I, J)=A \otimes^{\gamma} J+I \otimes^{\gamma} B$. Clearly $K_{A} \supseteq I$ and $K_{B} \supseteq J$. Also, for $a \in K_{A}$, if $I^{\prime}$ denotes the closed ideal generated by $a$ in $A$, then $I^{\prime} \otimes^{\gamma} B \subseteq K$. So, $I^{\prime} \in I d^{\prime}(A)$ and by Lemma 4.2. $I^{\prime} \subseteq I$, giving that $K_{A} \subseteq I$ and hence $K_{A}=I$. Similarly, we can see that $K_{B}=J$. As a consequence, $\Phi$ is injective on $\operatorname{Id}^{\prime}(A) \times \operatorname{Id}^{\prime}(B)$.

It now suffices to show that $\Psi$ is $\tau_{w}$-continuous. Consider a subbasic open set $U(I) \times U(J)$ of $\operatorname{Id}(A) \times I d(B)$, where $I \in I d(A), J \in I d(B)$. For any $K \in I d\left(A \otimes^{\gamma} B\right)$,

$$
\begin{aligned}
K \in \Psi^{-1}(U(I) \times U(J)) & \Longleftrightarrow K_{A} \in U(I) \quad \& \quad K_{B} \in U(J) \\
& \Longleftrightarrow K_{A} \nsupseteq I \quad \& \quad K_{B} \nsupseteq J \\
& \Longleftrightarrow K \nsupseteq I \otimes^{\gamma} B \quad \& \quad K \nsupseteq A \otimes^{\gamma} J \\
& \Longleftrightarrow K \in U\left(I \otimes^{\gamma} B\right) \cap U\left(A \otimes^{\gamma} J\right)
\end{aligned}
$$

Thus $\Psi^{-1}(U(I) \times U(J))=U\left(I \otimes^{\gamma} B\right) \cap U\left(A \otimes^{\gamma} J\right)$ and since the latter set is open in $\operatorname{Id}\left(A \otimes^{\gamma} B\right)$, this proves our claim.

We now show that $\Phi\left(I d^{\prime}(A) \times I d^{\prime}(B)\right)$ is dense in $I d^{\prime}\left(A \otimes^{\gamma} B\right)$. For this, consider a $K$ in $I d^{\prime}\left(A \otimes^{\gamma} B\right)$ and let $U$ be a basic open set in $I d^{\prime}\left(A \otimes^{\gamma} B\right)$ containing $K$. Then $U=\cap_{i=1}^{n} U\left(P_{i}\right)$ for some $P_{i} \in I d^{\prime}\left(A \otimes^{\gamma} B\right)$. Here, $K \in U\left(P_{i}\right)$, that is, $P_{i} \nsubseteq K$ for all $1 \leq i \leq n$. Now for each $i$, note that $A \otimes^{\gamma} K_{B}+K_{A} \otimes^{\gamma} B \subseteq K$; so $P_{i} \nsubseteq A \otimes^{\gamma} K_{B}$ and $P_{i} \nsubseteq K_{A} \otimes^{\gamma} B$, since $P_{i} \nsubseteq K$. This further implies that $P_{i} \nsubseteq \Phi\left(0, K_{B}\right)$ and $P_{i} \nsubseteq \Phi\left(K_{A}, 0\right)$ so that $\Phi\left(0, K_{B}\right) \in U\left(P_{i}\right)$ and $\Phi\left(K_{A}, 0\right) \in U\left(P_{i}\right)$ for all $1 \leq i \leq n$. Thus $U \cap \operatorname{Im}(\Phi) \neq \phi$ and hence image of $\Phi$ is dense in $I^{\prime}\left(A \otimes^{\gamma} B\right)$.

We now briefly recall hull-kernel topology, without details. Let $A$ be a Banach algebra. For each $E \subseteq \operatorname{Prime}(A)$, the set of all proper closed prime ideals of $A$, one associates a closed ideal, called kernel of $E$, given by $k(E)=\bigcap_{P \in E} P$. Also, for each $M \subseteq A$, hull of $M$ is defined as

$$
h_{A}(M)=\{P \in \operatorname{Prime}(A): P \supseteq M\} .
$$

Equip Prime $(A)$ with the hull-kernel topology (hk-topology, in short), where for $E \subseteq$ Prime $(A)$, its closure turns out to satisfy $\bar{E}=h(k(E))$, which can be taken as the definition of closure for our purpose - for details, see [3] and references therein.

As mentioned above, it is a fact that for any Banach algebra $A$, the $\tau_{w}$-topology coincides with the hull-kernel topolgy on $\mathcal{M}(A)$ - see [3]. The above homeomorphism restricts well to maximal and maximal modular ideals.

THEOREM 4.4. Let $A$ and $B$ be $C^{*}$-algebras. Then, the restriction of $\Phi$ to $\mathcal{M}(A) \times \mathcal{M}(B)$ is a homeomorphism onto $\mathcal{M}\left(A \otimes^{\gamma} B\right)$ with respect to the hull-kernel topology. Furthermore, $\Phi$ maps $\mathcal{M}_{m}(A) \times \mathcal{M}_{m}(B)$ homeomorphically onto $\mathcal{M}_{m}\left(A \otimes^{\gamma} B\right)$, as well.

Proof. By Equation (4.1), Theorem 3.21 and Theorem 3.22, $\Phi$ maps $\mathcal{M}(A) \times \mathcal{M}(B)$ (resp., $\left.\mathcal{M}_{m}(A) \times \mathcal{M}_{m}(B)\right)$ bijectively onto $\mathcal{M}\left(A \otimes^{\gamma} B\right)$ (resp., $\left.\mathcal{M}_{m}\left(A \otimes^{\gamma} B\right)\right)$.

Since $\Phi$ is continuous (being $\tau_{w}$-continuous), it just remains to show that $\Phi$ is a closed map with respect to the product $h k$-topology on $\mathcal{M}(A) \times \mathcal{M}(B)$ and the $h k$-topology on $\mathcal{M}\left(A \otimes^{\gamma} B\right)$. First of all note that any closed set in $\mathcal{M}(A) \times \mathcal{M}(B)$ is of the form

$$
\cap_{\alpha}\left(\left(F_{A}^{\alpha} \times \mathcal{M}(B)\right) \cup\left(\mathcal{M}(A) \times F_{B}^{\alpha}\right)\right),
$$

where $F_{C}^{\alpha}$ is closed in $\mathcal{M}(C)$ for $C=A, B$. Also, since $\Phi$ is injective, we have

$$
\Phi\left(\cap_{\alpha}\left(\left(F_{A}^{\alpha} \times \mathcal{M}(B)\right) \cup\left(\mathcal{M}(A) \times F_{B}^{\alpha}\right)\right)\right)=\cap_{\alpha}\left(\Phi\left(F_{A}^{\alpha} \times \mathcal{M}(B)\right) \cup \Phi\left(\mathcal{M}(A) \times F_{B}^{\alpha}\right)\right) .
$$


Thus, it is sufficient to prove that for any closed set $F_{A}$ of $\mathcal{M}(A), X:=\Phi\left(F_{A} \times \mathcal{M}(B)\right)$ is closed in $\mathcal{M}\left(A \otimes^{\gamma} B\right)$. We have to show that $h(k(X)) \subseteq X$. Let $P \in \mathcal{M}\left(A \otimes^{\gamma} B\right)$ be such that $k(X) \subseteq P$. Let, if possible, $P \notin X$. Since $P=A \otimes^{\gamma} J+I \otimes^{\gamma} B=\operatorname{ker}\left(\pi_{I} \otimes^{\gamma} \pi_{J}\right)$, for some $I \in \mathcal{M}(A), J \in \mathcal{M}(B)$, it follows that $I \notin F_{1}$. But $F_{A}$ is closed in $h k$-topology, thus $k\left(F_{A}\right) \nsubseteq I$. Let $a \in k\left(F_{A}\right) \backslash I$ and fix $b \notin J$. Note that $(a+I) \otimes(b+J) \neq 0$ which gives that $a \otimes b \notin \operatorname{ker}\left(\pi_{I} \otimes^{\gamma} \pi_{J}\right)=P$. On the other hand, consider any $K:=\Phi(L \times M) \in X$, where $L \in F_{A}$ and $M \in \mathcal{M}(B)$. Since $a \in k\left(F_{A}\right) \subseteq L$ we have $(a+L) \otimes(b+M)=0$. Thus $a \otimes b \in \operatorname{ker}\left(\pi_{L} \otimes^{\gamma} \pi_{M}\right)=K$ and this is true for all $K \in X$. So, $a \otimes b \in k(X) \subseteq P$, which gives a contradiction.

\section{Center of $A \otimes^{\gamma} B$}

For algebras $A$ and $B$ with centers $\mathcal{Z}(A)$ and $\mathcal{Z}(B)$, respectively, one can easily check that there is a canonical algebra isomorphism between $\mathcal{Z}(A \otimes B)$ and $\mathcal{Z}(A) \otimes \mathcal{Z}(B)$. For any two $C^{*}$-algebras $A$ and $B$ and any $C^{*}$-norm $\|\cdot\|_{\alpha}$, it is known that the above isomorphism extends to an isometric $*$-isomorphism from $\mathcal{Z}\left(A \otimes^{\alpha} B\right)$ onto $\mathcal{Z}(A) \otimes^{\alpha} \mathcal{Z}(B)$ - see [2. Making explicit use of injectivity of $\otimes^{h}$, the above natural map also extends to an isometric algebra isomorphism from $\mathcal{Z}\left(A \otimes^{h} B\right)$ onto $\mathcal{Z}(A) \otimes^{h} \mathcal{Z}(B)$ - see [1]; and for $\widehat{\otimes}$, it extends to an algebraic $*$-isomorphism (not necessarily isometric) from $\mathcal{Z}(A \widehat{\otimes} B)$ onto $\mathcal{Z}(A) \widehat{\otimes} \mathcal{Z}(B)$ - see [15]. With the kind of partial injectivity for $\otimes^{\gamma}$ established in Section 2, their analogue for $\otimes^{\gamma}$ is quite satisfying.

Theorem 5.1. For $C^{*}$-algebras $A$ and $B, \mathcal{Z}\left(A \otimes^{\gamma} B\right)=\mathcal{Z}(A) \otimes^{\gamma} \mathcal{Z}(B)$.

Proof. Since $\mathcal{Z}(A \otimes B) \subseteq \mathcal{Z}\left(A \otimes^{\gamma} B\right)$, consider the identity function from $\mathcal{Z}(A) \otimes \mathcal{Z}(B)$ into $\mathcal{Z}\left(A \otimes^{\gamma} B\right)$. By Theorem 2.6. $\mathcal{Z}(A) \otimes^{\gamma} \mathcal{Z}(B)$ can be considered as a $*$-subalgebra of $A \otimes^{\gamma} B$, so that for any $u \in \mathcal{Z}(A) \otimes \mathcal{Z}(B),\|u\|_{\mathcal{Z}(A) \otimes \gamma \mathcal{Z}(B)}=\|u\|_{A \otimes \gamma B}$. Thus, the identity function extends uniquely to an isometric $*$-homomorphism, say, $\theta$ from $\mathcal{Z}(A) \otimes^{\gamma} \mathcal{Z}(B)$ into $\mathcal{Z}\left(A \otimes^{\gamma} B\right)$. It only remains to show that $\theta$ is surjective.

Let $z \in \mathcal{Z}\left(A \otimes^{\gamma} B\right)$. Consider $i: A \otimes^{\gamma} B \rightarrow A \otimes^{h} B$, the canonical injective homomorphism as in Proposition 3.2. It is easily seen that $i(z) x=x i(z)$ for all $x \in \mathcal{Z}(A) \otimes \mathcal{Z}(B)$; so that $i(z) \in \mathcal{Z}(A) \otimes^{h} \mathcal{Z}(B)$, and by 11, we have $\mathcal{Z}\left(A \otimes^{h} B\right)=\mathcal{Z}(A) \otimes^{h} \mathcal{Z}(B)$. Now, let $i^{\prime}: \mathcal{Z}(A) \otimes^{\gamma}$ $\mathcal{Z}(B) \rightarrow \mathcal{Z}(A) \otimes^{h} \mathcal{Z}(B)$ be the canonical injective homomorphism (like the map $i$ ). Then, the following diagram

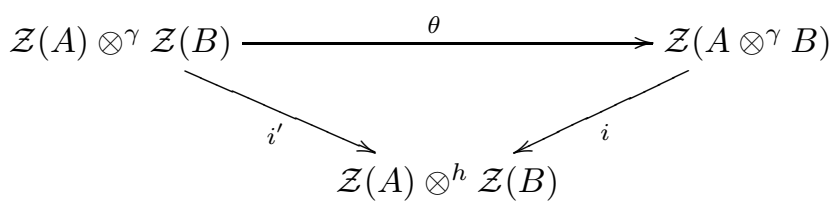

commutates because $i \circ \theta=i^{\prime}$ on $\mathcal{Z}(A) \otimes \mathcal{Z}(B)$ and all the three maps are continuous.

Note that, the map $i^{\prime}$ is surjective as well. To see this, consider an element $z^{\prime} \in \mathcal{Z}(A) \otimes^{h} \mathcal{Z}(B)$ and fix a sequence $\left\{z_{n}\right\} \subseteq \mathcal{Z}(A) \otimes \mathcal{Z}(B)$ such that $\left\|z_{n}-z^{\prime}\right\|_{h} \rightarrow 0$. By Grothendieck inequality for commutative $C^{*}$-algebras (see [26]), we have

$$
\|x\|_{\gamma} \leq K_{G}\|x\|_{h} \text { for all } x \in \mathcal{Z}(A) \otimes \mathcal{Z}(B),
$$

$K_{G}$ being the Grothendieck constant. Thus, the sequence $\left\{z_{n}\right\}$ is Cauchy with respect to $\|\cdot\|_{\gamma}$ and converges to some $z^{\prime \prime}$ in $\mathcal{Z}(A) \otimes^{\gamma} \mathcal{Z}(B)$. This shows that $\left\{z_{n}=i^{\prime}\left(z_{n}\right)\right\}$ converges to $z^{\prime}$ as well as to $z^{\prime \prime}$ in $\mathcal{Z}(A) \otimes^{h} \mathcal{Z}(B)$. So, $i^{\prime}\left(z^{\prime \prime}\right)=z^{\prime}$ and $i^{\prime}$ is surjective.

Thus, for above $i(z)$ in $\mathcal{Z}(A) \otimes^{h} \mathcal{Z}(B)$, there exists some $w \in \mathcal{Z}(A) \otimes^{\gamma} \mathcal{Z}(B)$ such that $i(z)=i^{\prime}(w)=i(\theta(w))$. Since $i$ is injective, $z=\theta(w)$, so that $\theta$ is surjective and we are done. 
It would be interesting to provide an answer to the following:

QUESTION. Is there any relationship, as above, between the center of $A \otimes^{\gamma} B$ and $\mathcal{Z}(A) \otimes^{\gamma} \mathcal{Z}(B)$ for Banach algebras $A$ and $B$ ?

\section{REFERENCES}

[1] S. D. Allen, A. M. Sinclair and R. R. Smith, The ideal structure of the Haagerup tensor product of $C^{*}-$ algebras, J. Reine Angew. Math., 442, 1993, 111 - 148.

[2] R. J. Archbold, On the centre of a tensor product of $C^{*}$-algebras, J. London Math. Soc., 10, 1975, 257 262.

[3] ......... Topologies for primal ideals, J. London Math. Soc.(2), 37, 1987, $524-542$.

[4] R. J. Archbold, D. W. B. Somerset, E. Kaniuth and G. Schlichting, Ideal spaces of the Haagerup tensor product of $C^{*}$-algebras, International J. Math., 8 (1), 1997, 1 - 29.

[5] E. Blanchard and E. Kirchberg, Non-simple purely infinite $C^{*}$-algebras: the Hausdorff case, J. Funct. Anal., 207, 2004, $461-513$.

[6] J. Diestel, A. M. Peralta and D. Puglisi, Sequential w-right continuity and summing operators, Math. Nachr., 284, 2011, $664-680$.

[7] P. G. Dixon, Non-closed sums of closed ideals in Banach algebras, Proc. Amer. Math. Soc., 128 (12), 2000, $3647-3654$.

[8] E. G. Effors and Z.-J. Ruan, Operator Spaces, LMS Monographs New Series 23, Oxford University Press, 2000.

[9] B. R. Gelbaum, Tensor products of Banach algebras, Canad. J. Math, 11, 1959, 297 - 310.

[10] ........., Note on the tensor product of Banach algebras, Proc. Amer. Math. Soc., 12 (5), 1961, 750 757.

[11] ........., Tensor products of Banach algebras II, Proc. Amer. Math. Soc., 25(3), 1970, 470 - 474.

[12] V. P. Gupta and R. Jain, On closed Lie ideals of certain tensor products of $C^{*}$-algebras, Math. Nachr., 2017. DOI: $10.1002 /$ mana.201700009.

[13] U. Haagerup, The Grothendieck inequality for bilinear forms on $C^{*}$-algebras, Adv. Math., 56, 1985,93 116.

[14] R. Jain, Operator space tensor products of $C^{*}$-algebras and their ideal structures, Doctoral Thesis, University of Delhi, 2012.

[15] R. Jain and A. Kumar, Operator space tensor products of $C^{*}$-algebras, Math. Z., 260, 2008, $805-811$.

[16]..$\ldots \ldots \ldots$, Ideals in operator space projective tensor product of $C^{*}$-algebras, J. Aust. Math. Soc., 91, $2011,275-288$

[17] ........., The operator space projective tensor product: Embedding into the second dual and ideal structure, Proc. Edin. Math. Soc., 57, 2014, 505 - 519.

[18] ........., Spectral synthesis for the operator space projective tensor product of $C^{*}$-algebras, Bull. Malays. Math. Scie. Soc. (2), 36 (4), 2013, 855 - 864.

[19] A. Kumar, Operator space projective tensor product of $C^{*}$-algebras, Math. Z., 237, 2001, 211 - 217.

[20] A. Kumar and V. Rajpal, Symmetry and quasi-centrality of operator space projective tensor product, Arch. Math., 99, 2012, 519 - 529.

[21] ........., Projective tensor products of $C^{*}$-algebras, Adv. Pure Math., 4, 2014, 176 - 188.

[22] S. Kaijser and A. M. Sinclair, Projective tensor products of $C^{*}$-algebras, Math. Scand., 55, 1984, 161 - 187.

[23] K. B. Laursen, Maximal two sided ideals in tensor products of Banach algebras, Proc. Amer. Math. Soc., 25 (3), 1970, $475-480$.

[24] A. J. Lazar, The space of ideals in the minimal tensor products of $C^{*}$-algebras, Math. Proc. Camb. Phil. Soc., 148, 2010, $243-252$.

[25] R. J. Loy. Identities in tensor product of Banach algebras, Bull. Aust. Math. Soc., 2, 1970, 253 - 260.

[26] G. Pisier. Factorization of linear operators and geometry of Banach spaces. CBMS Regional Conf. Series in Math, 60, Amer. Math. Soc., Providence, RI, 1986.

[27] R. A. Ryan, Introduction to tensor products of Banach spaces, Springer, 2002.

[28] M. Takesaki, Theory of Operator Algebras I, Springer-Verlag, 1979.

[29] J. Tomiyama, Primitive ideals in tensor products of Banach algebras, Math. Scand., 30, 1972, 257 - 262.

School of Physical Sciences,, Jawaharlal Nehru University, New Delhi-110067, INDiA.

E-mail address: vedgupta@mail.jnu.ac.in

Department of Mathematics, University of Delhi, Delhi-110007, indiA.

E-mail address: rjain@maths.du.ac.in 